\section{Action video game modifies visual selective attention}

\section{Shawn Green \& Daphne Bavelier}

Department of Brain and Cognitive Sciences, Center for Visual Science, University of Rochester, Rochester, New York 14627, USA

As video-game playing has become a ubiquitous activity in today's society, it is worth considering its potential consequences on perceptual and motor skills. It is well known that exposing an organism to an altered visual environment often results in modification of the visual system of the organism. The field of perceptual learning provides many examples of training-induced increases in performance. But perceptual learning, when it occurs, tends to be specific to the trained task; that is, generalization to new tasks is rarely found ${ }^{1-10}$. Here we show, by contrast, that action-video-game playing is capable of altering a range of visual skills. Four experiments establish changes in different aspects of visual attention in habitual video-game players as compared with non-video-game players. In a fifth experiment, non-players trained on an action video game show marked improvement from their pre-training abilities, thereby establishing the role of playing in this effect.

We first used the flanker compatibility effect, a standard experimental paradigm in attentional studies, to determine whether video-game playing produces an overall increase in attentional capacity $^{11}$. This task measures the effect of a to-be-ignored distractor on a target task. The size of this distractor effect has been proposed to provide an index of 'left-over' attentional resources. In particular, it has been reported ${ }^{12}$ that the distractor effect is large when the target task is easy, but small when the target task is made difficult by increasing the number of filler shapes in the ring (Fig. 1a). The currently accepted basis for this finding is that when the target task is easy, spare attentional resources 'spill over' to the distractor, processing it to some extent and thereby influencing target processing. As the target task becomes more difficult, fewer attentional resources remain to process the extraneous distractor. Irrelevant processing is only truly prevented once the target task becomes so difficult that all available resources are devoted to the target task, leaving none to the distractor.

This task therefore affords an opportunity to test the hypothesis that video-game playing increases the capacity of the visual attentional system. If video-game players (VGPs) do indeed have a greater attentional capacity, they should exhaust their visual attention resources more slowly than non-video-game players (NVGPs) as the target task becomes more difficult. As predicted by the hypothesis of a greater capacity in their population, VGPs showed an overall distractor effect that remained even when the target task was difficult (Fig. 1b). Thus, at difficulty levels where NVGPs have long depleted their attentional resources, VGPs possess sufficient resources to perform the target task, with resources still remaining to spill over to the distractors. These results indicate that VGPs possess enhanced attentional capacity.

We confirmed this increase in capacity more directly through the use of an enumeration task. When asked to report how many squares are presented in a briefly flashed display, normal subject performance seems to be best captured by two distinct processes: an 'automatic' operation for small numbers of targets in which performance is rapid, accurate and independent of number (subitizing); and a slower, serial counting process for larger numbers of items ${ }^{13}$. The number of items that can be subitized gives an estimate of the number of items that can be attended at once $^{14-16}$. VGPs could subitize more items than NVGPs (4.9 versus 3.3 items; population effect, $P<0.01$; Fig. 2). Thus,
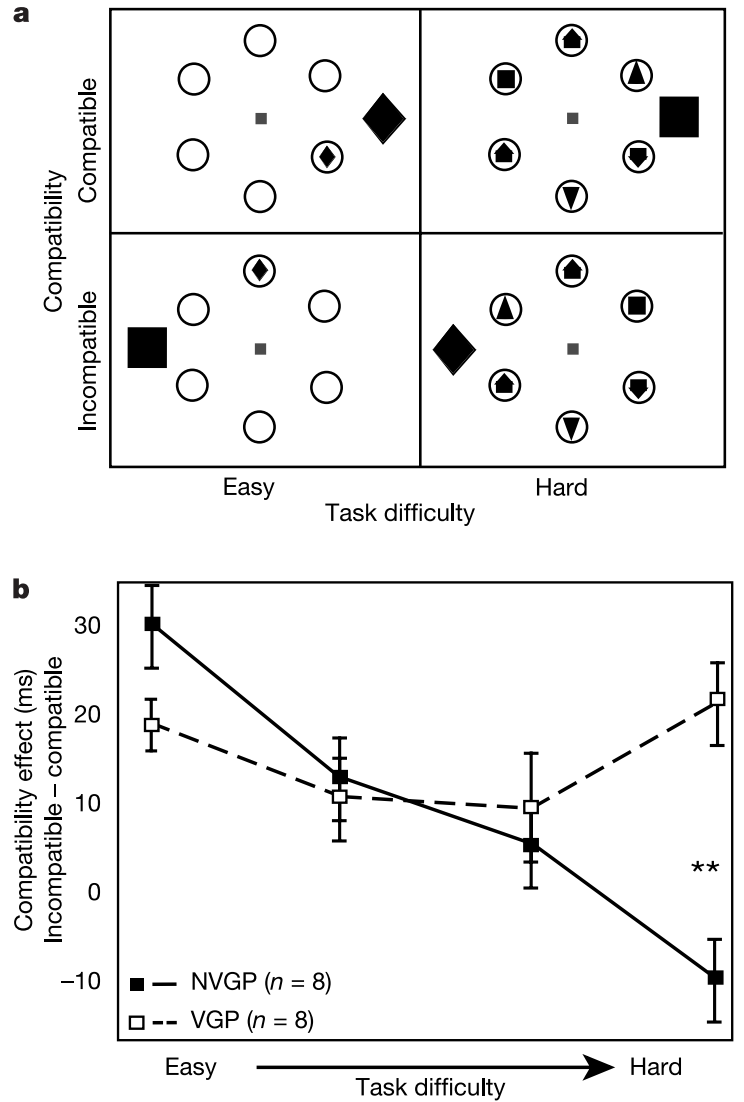

Figure 1 Measure of attentional resources. a, Flanker compatibility task. Participants were asked to decide whether a square or a diamond appeared within one of the six rings (target task), while ignoring a distractor shape presented outside the rings. The distractor shape could be either compatible (the same as the target shape) or incompatible (the alternative target shape). Of interest was the difference in target processing speed between compatible and incompatible trials. This difference, also called the 'compatibility effect', measures the attentional resources available to the participant. By inserting no, one, three or five extra shapes into the rings, the difficulty of the target task was manipulated to test the possibility that VGPs show compatibility effects at task difficulties for which attentional resources are usually exhausted in NVGPs. b. Flanker compatibility effect. As previously reported ${ }^{12}$, the compatibility effect (and spare attentional resources) decreased with task difficulty in NVGPs (task difficulty $\times$ compatibility, $P<0.001$ ). In VGPS, by contrast, the compatibility effect remained as the target task was made more difficult (population $\times$ task difficulty $\times$ compatibility, $P<0.003$ ). As a result, VGPs

showed a significantly greater compatibility effect than NVGPs when the target task was difficult, indicating greater attentional resources in VGPS $\left.{ }^{\star \star} P<0.01\right)$. Error bars denote s.e.m.

video-game playing enhances the number of visual items that can be unerringly apprehended.

These two experiments indicated that video-game playing enhances attentional capacity in an area well within the 'training' (video-game playing) zone $\left(0-5^{\circ}\right.$ from fixation); however, it was unclear whether video-game playing also facilitates processing outside the training range. To test this possibility, we compared the distribution of visual attention in VGPs and NVGPs at three locations, one well within the training range $\left(10^{\circ}\right)$, one at its boundary $\left(20^{\circ}\right)$ and one well outside the training range $\left(30^{\circ}\right.$; all VGPs reported playing in a field that extends no more than $18^{\circ}$ from fixation). We adapted the 'useful field of view' task (refs 17-19 and Fig. 3a), which measures participants' ability to locate a target amongst distractors. This measure does not correlate well with standard tests of visual acuity but rather provides a measure of attentional resources and their spatial distribution ${ }^{17}$. VGPs far outperformed NVGPs at all eccentricities (Fig. 3b), distractor levels and distractor-eccentricity pairs. These results indicate an 


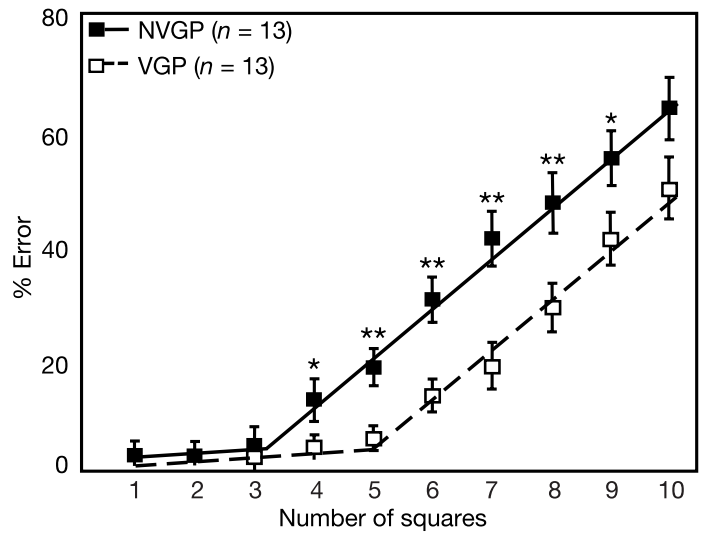

Figure 2 Enumeration performance. When asked to report the number of squares briefly flashed, VGPs were able to apprehend more items at once than were NVGPs (4.9 versus 3.3). Overall, VGPs were significantly more accurate than NVGPs ( $78 \%$ versus $65 \%$, $P<0.003$ ) and, as expected, this difference only emerged for numbers above the subitizing range of NVGPS. Error bars denote s.e.m. $\left({ }^{\star} P<0.05,{ }^{*} P<0.01\right)$.

enhanced allocation of spatial attention over the visual field, even at untrained locations, in VGPs.

The three experiments described so far indicated that video-game playing enhances the capacity of visual attention and its spatial distribution. We next examined the temporal characteristics of visual attention and asked whether the pressure to act rapidly on several visual items, which is inherent to most action games, alters

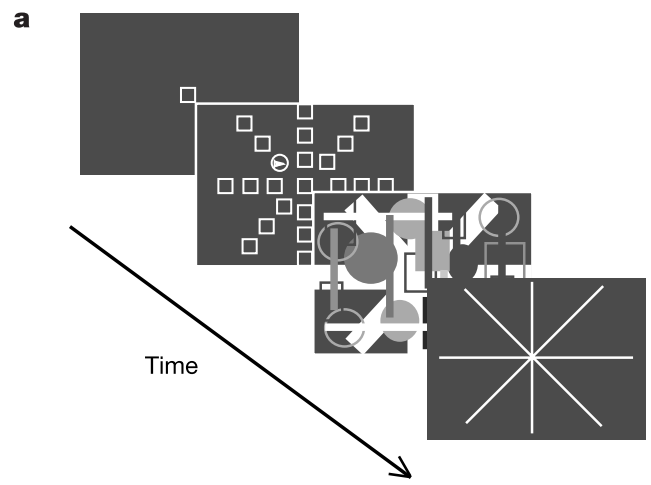

b

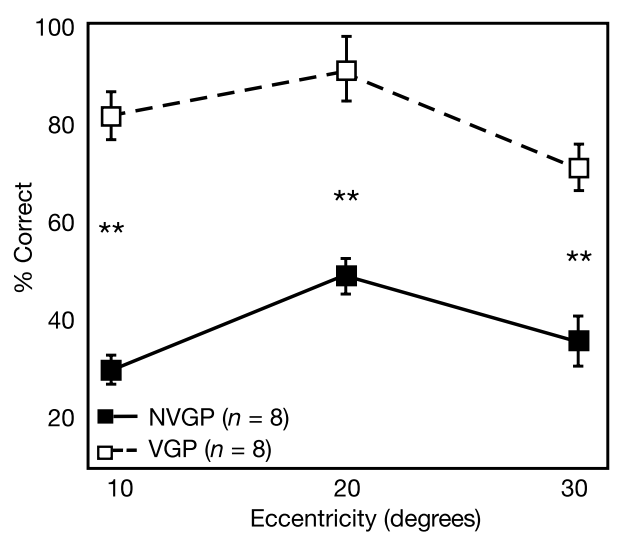

Figure $\mathbf{3}$ Measure of attention over space. a, Sequence of displays in the useful field of view task. Participants indicated the spoke on which the small target (triangle within circle) appeared. By presenting the target at different eccentricities, this task affords an opportunity to test the spatial distribution of visual attention. $\mathbf{b}$, Localization accuracy. VGPs showed large enhancements in localization ability at all eccentricities. The superiority of $\mathrm{VGPs}$ at $30^{\circ}$ indicates that the enhancement of spatial attention observed in this population is not limited to trained locations. Error bars denote s.e.m. $\left({ }^{\star \star} P<0.01\right)$. the ability to process items over time, particularly the ability to avoid 'bottlenecks' of attention that often occur in temporal processing. The experimental paradigm that we used to test the temporal aspects of visual attention is the attentional blink task (refs 20-22 and Fig. 4a). Attentional blink refers to the phenomena wherein subjects have difficulties reporting a second target when it appears a few hundreds of milliseconds after the onset of a first target. In our case, we used a variant of the attentional blink, in which participants were asked to identify a first target and then detect a second target. This task includes two distinct attentional bottlenecks. First is the attentional blink per se, which is the difficulty of processing a second target that comes $200-500 \mathrm{~ms}$ after the onset of the first one. Second is the cost of switching tasks between the first and second target (from identification to detection); unlike the attentional blink per se, this effect is most pronounced when the two targets are temporally adjacent and then decreases slowly as the time between the two targets increases. This attentional bottleneck is not specific to vision but rather appears amodal $^{21,23,24}$. Thus, by using an identification/detection attentional blink task, we could test whether the enhanced capacities after video-game training not only applied to a purely visual bottleneck but also generalized to an amodal one.

a
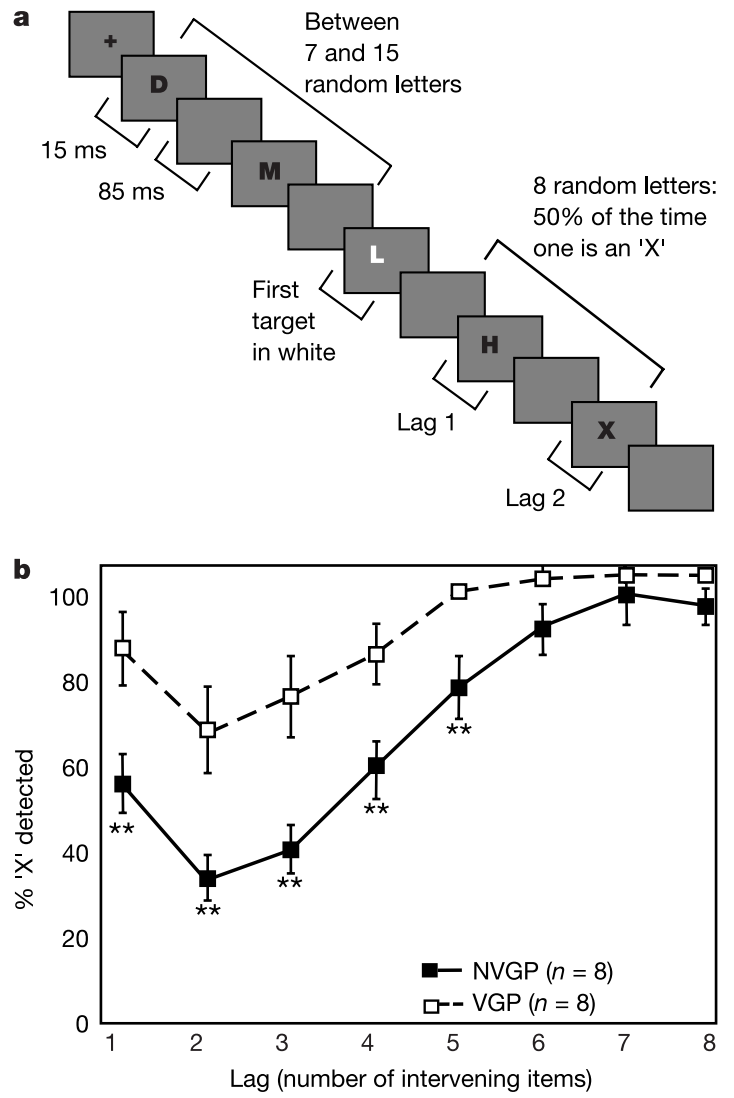

Figure 4 Measure of attention over time. a, Attentional blink task. Black letters were rapidly presented at fixation in a standard rapid, serial, visual presentation manner. At a random time in the stream, a white letter was presented (first target). After this first target, an ' $X$ ' (second target) was presented among the following letters $50 \%$ of the time. After the trial, the subject gave the identity of the first target and then indicated whether the ' $X$ ' was presented. Of interest is the performance of subjects on the ' $X$ ' detection task, given that they have correctly identified the white letter. $\mathbf{b}$, Attentional blink performance. At early lags, VGPs performed better (less blink) than NVGPs; as lag increased, the effect of the attentional bottlenecks decreased and, as expected, the two populations became comparable (lag by population $P<0.02$ ). Error bars denote s.e.m. Points without error bars indicate that the s.e.m. was smaller than the size of the square $\left({ }^{\star \star} P<0.01\right)$. 
VGPs outperformed NVGPs on second-target correct detections from lag 1 to lag 5, indicating less attentional blink (Fig. 4b). The finding of a population difference as early as lag 1 indicates that video-game training enhances task-switching abilities as well as decreasing the attentional blink. Thus, both the visual and amodal bottlenecks identified during temporal processing of visual information are reduced in VGPs. Clearly, these individuals have an increased ability to process information over time; however, whether this is due to faster target processing, such as faster selection and stabilization of information in memory, or to an increased ability to maintain several attentional windows in parallel, cannot be determined from our current data.

We also carried out a training experiment to alleviate concerns over the source of the VGP and NVGP population differences. By selecting VGPs, we may have selected individuals with inherently better attentional skills than NVGPs. The consequence of this potential split would be that VGPs, with their greater natural ability, did well at video games and so played often, whereas NVGPs, whose inherent abilities limited their success, avoided playing games as a result. Another possible confound was that, because of relatively superior visuo-motor coordination, VGPs were better able to perform the motor aspect of the tasks and thus had more spare resources to devote to the visually demanding part of the tasks.

To rule out these potential confounds, a group of NVGPs underwent action-video-game training, in which they were asked to play Medal of Honor (a game similar to those reported being played by
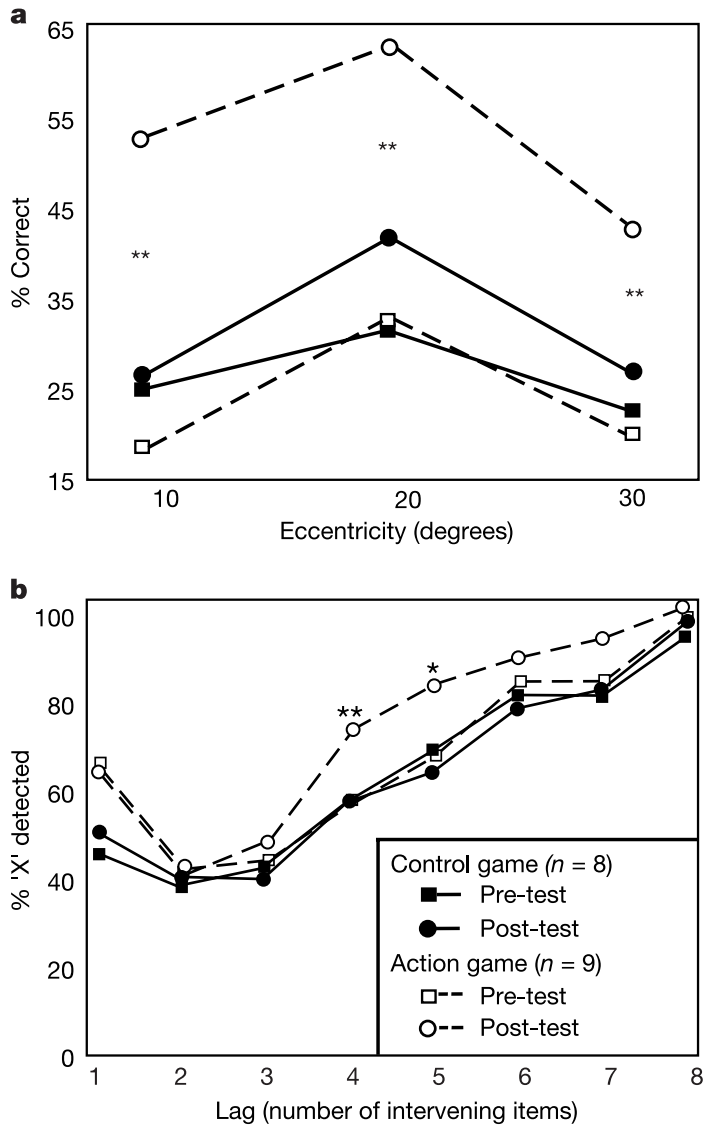

Figure 5 Performance before and after training. a, Training: useful field of view. At each eccentricity, the group trained on an action video game improved significantly more from their pre-test scores than did the control group trained on a non-action video game $\left({ }^{\star} P<0.01\right)$. b. Training: attentional blink. The group trained on an action video game recovered faster from the attentional blink than did the control group trained on a nonaction video game $\left({ }^{\star} P<0.05,{ }^{* *} P<0.01\right)$. the VGP population) for $1 \mathrm{~h}$ per day for 10 consecutive days. A control group was trained, over the same time span, on the game Tetris. This game contains a challenging visuo-motor component but, whereas action games require that attention is distributed and/or switched around the field, Tetris demands focus on one object at a time. Tetris, therefore, would not be expected to change the aspects of visual attention described above and thus affords an excellent control for monitoring improvement due to enhanced visuo-manual expertise and test-retest improvements.

Before and after training we tested each group on the enumeration, useful-field-of-view and attentional-blink experiments. The training was successful as all participants improved their scores on the video game on which they were trained. Notably, action-game training led to greater performance improvement than did the control game on all three experimental tasks. Enumeration increased by 1.7 items in individuals trained on action games, whereas the control group showed no improvement (population by improvement, $P<0.05)$. Training on action video games enhanced the useful field of view and lead to faster recovery from the attentional blink (Fig. 5). In addition, we found that the improvement in these attentional measures was marginally correlated with improvement in video-game playing (adjusted $r^{2}=0.43$, $P=0.13$; see Supplementary Information). Thus, 10 days of training on an action game is sufficient to increase the capacity of visual attention, its spatial distribution and its temporal resolution.

By forcing players to simultaneously juggle a number of varied tasks (detect new enemies, track existing enemies and avoid getting hurt, among others), action-video-game playing pushes the limits of three rather different aspects of visual attention. It leads to detectable effects on new tasks and at untrained locations after only 10 days of training. Therefore, although video-game playing may seem to be rather mindless, it is capable of radically altering visual attentional processing. There are several ways by which video-game training could lead to such enhancements. Changes in known attentional bottlenecks is certainly a possibility; however, speeded perceptual processes and/or better management of several tasks at the central executive level are also likely to contribute. It will be for future studies of the effect of video-game practice to determine the relative contribution of these different factors to skill learning.

\section{Methods Participants}

Subjects were aged between 18 and 23 years. The VGPs had played action video games on at least 4 days per week for a minimum of $1 \mathrm{~h}$ per day for the previous 6 months. The games included Grand Theft Auto3, Half-Life, Counter-Strike, Crazy Taxi, Team Fortress Classic, 007, Spider-Man, Halo, Marvel vs Capcom, Roguespeare and Super Mario Cart. The NVGPs had little, and preferably no, video-game usage in the past 6 months. Experiments 1-4 included only males; in experiment 5 , both male and female NVGPs underwent training.

\section{Apparatus, stimuli and procedure}

For experiment 1-flanker compatibility (Fig. 1) - the apparatus, stimulus, procedure and data analysis have been described ${ }^{25}$.

For experiment 2-enumeration (Fig. 2) - the stimuli consisted of a random number (between 1 and 12) of white squares (each subtending $0.5^{\circ} \times 0.5^{\circ}$ ) presented for $50 \mathrm{~ms}$ within an invisible $5^{\circ} \times 5^{\circ}$ square centred on the screen. Subjects were asked to type the number of squares that they believed were presented; no feedback was provided. We scored the percentage of trials in which participants correctly reported the number of squares presented. Only data from trials with 1-10 squares are presented, as trials with 11 and 12 squares were excessively difficult for all populations. To estimate the point at which subjects switched from subitizing to counting, we modelled the data of each subject with a bilinear curve. The first line was set to have an intercept of $(0,0)$ and was expected to have a rather shallow slope (subitizing range); the second line was expected to have a steeper slope (counting). The best bilinear fit was found by minimizing the least-square error. The point at which the two lines met was then computed and considered to be the point where subitizing switches to counting.

For experiment 3-useful field of view (Fig. 3) - the stimuli and procedure were similar to those used previously ${ }^{17}$, except that the stimulus size and presentation time were both decreased to account for the increased ability of comparatively younger subjects (target stimulus was a filled triangle within a $3^{\circ} \times 3^{\circ}$ circle outline; distracting squares subtended $4^{\circ} \times 4^{\circ}$, no central task). Separate timings were used to ensure equal difficulty at each 
eccentricity: $6 \mathrm{~ms}$ for targets presented at $10^{\circ}$; and $12 \mathrm{~ms}$ for targets present at either $20^{\circ}$ or $30^{\circ}$

For experiment 4-attentional blink (Fig. 4) - the stimuli and procedure described previously ${ }^{20}$ were used except that eight lags instead of nine, and eight trials per condition instead of ten, were used. VGPs had almost significantly higher-second-target detection accuracy in the control condition (detecting the $\mathrm{X}$ in the absence of a first target, $95.6 \%$ to $87.9 \%, P=0.061$ ). Conservatively, all analyses were done on the difference between the variable of interest and the baseline detection condition to eliminate different baseline abilities as a potential confound in interpreting the depth of the attentional blink between populations.

For experiment 5-training (Fig. 5) -all subjects were tested on the useful-field-ofview, attentional-blink and enumeration tasks (in this order) before training; they then underwent training for $1 \mathrm{~h}$ per day for 10 days with one of two possible kinds of video game. After training they were retested on the same three tasks (in the same order). The experimental group trained on Medal of Honor: Allied Assault (Electronic Arts, 2002). This game simulates Second World War combat situations and was chosen to be similar to those played by our VGPs. It has a relatively simple interface, uses first-person point of view and requires effective monitoring of the whole visual field (extent from fixation about $13^{\circ}$ (height) $\times 16^{\circ}$ (width) ). Subjects played the game straight through for the first 8 days On days 9 and 10, they returned to the beginning of the game to quantitatively measure their improvement (comparing performance over mission 1 during their first and second playings). In all available measures of improvement taken (accuracy and deaths to complete mission), subjects improved their performance after training (accuracy, 17\% improvement; deaths to complete, $42 \%$ improvement)

For the control group $(n=8)$, Tetris (Original Tetris, AcademySoft Elorgwhich, 1987; Tengen, 1988) was displayed to cover the entire extent of the screen. Because Tetris adds graphics on the side of the screen, however, the effective game area extended $13^{\circ}$ (height) $\times 9^{\circ}$ (width) from fixation. This game was selected to control for the effect of improved visuo-motor coordination, while putting few demands on the processing of several objects at once. Accordingly, the version of Tetris on which subjects were trained had the preview block option turned off. In the two measures of improvement available (high score, maximum level reached), all subjects improved after training (high score, 71\% improvement; mean level improvement, 67\% improvement).

Received 4 December 2002; accepted 8 April 2003; doi:10.1038/nature01647.

1. Sagi, D. \& Tanne, D. Perceptual learning: learning to see. Curr. Opin. Neurobiol. 4, 195-199 (1994).

2. Ball, K. \& Sekuler, R. A specific and enduring improvement in visual motion discrimination. Science 218, 697-698 (1982).

Fiorentini, A. \& Berardi, N. Perceptual learning specific for orientation and spatial frequency. Nature 287, 43-44 (1980).

4. Karni, A. \& Sagi, D. Where practice makes perfect in texture discrimination: evidence for primary visual cortex plasticity. Proc. Natl Acad. Sci. USA 88, 4966-4970 (1991)

5. Poggio, T., Fahle, M. \& Edelman, S. Fast perceptual learning in visual hyperacuity. Science $\mathbf{2 5 6}$ 1018-1021 (1992)

6. Fahle, M. \& Edelman, S. Long-term learning in vernier acuity: effects of stimulus orientation, range, and of feedback. Vision Res. 33, 397-412 (1993).

7. Ramachandran, V. S. \& Braddick, O. Orientation-specific learning in stereopsis. Perception 2, 371-376 (1973).

8. Ahissar, M., Laiwand, R., Kozminsky, G. \& Hochstein, S. Learning pop-out detection: building representations for conflicting target-distractor relationship. Vision Res. 38, 3095-3107 (1998).

9. Ahissar, M. \& Hochstein, S. The spread of attention and learning in feature search: effects of target distribution and task difficulty. Vision Res. 40, 1349-1364 (2000).

10. Sirenteanu, R. \& Regina, R. Perceptual learning in visual search generalizes over task locations and eyes. Vision Res. 40, 2925-2949 (2000).

11. Eriksen, B. A. \& Eriksen, C. W. Effects of noise letters upon the identification of a target letter in nonsearch task. Percept. Psychophys. 16, 143-149 (1974).

12. Lavie, N. \& Cox, S. On the efficiency of visual selective attention: efficient visual search leads to inefficient distractor rejection. Psychol. Sci. 8, 395-398 (1997).

13. Kaufman, E., Lord, M., Reese, T. \& Volkman, J. The discrimination of visual number. Am. J. Psychol. 62, 498-525 (1949)

14. Trick, L. M. \& Pylyshyn, Z. W. What enumeration studies can show us about spatial attention evidence for limited capacity preattentive processing. J. Exp. Psychol. Human Percept. Perform. 19, 331-351 (1993).

15. Trick, L. M. \& Pylyshyn, Z. W. Why are small and large numbers enumerated differently? A limitedcapacity preattentive stage in vision. Psychol. Rev. 101, 80-102 (1994).

16. Tuholski, S. W., Engle, R. W. \& Baylis, G. C. Individual differences in working memory capacity and enumeration. Mem. Cogn. 29, 484-492 (2001).

17. Ball, K. K., Beard, B. L., Roenker, D. L., Miller, R. L. \& Griggs, D. S. Age and visual search: expanding the useful field of view. J. Optic. Soc. Am. 5, 2210-2219 (1988)

18. Myers, R. S., Ball, K. K., Kalina, T. D., Roth, D. L. \& Goode, K. T. Relation of useful field of view and other screening tests to on-road driving performance. Percept. Mot. Skills 91, 279-290 (2000).

19. Ball, K. K., Owsley, C., Sloane, M., Roenker, D. \& Bruni, J. Visual attention problems as a predictor of vehicle crashes in older drivers. Invest. Ophthalmol. 34, 3110-3123 (1993).

20. Raymond, J. E., Shapiro, K. L. \& Arnell, K. M. Temporary suppression of visual processing in an RSVP task: an attentional blink? J. Exp. Psychol. Human Percept. Perform. 18, 849-860 (1992)

21. Chun, M. M. \& Potter, M. C. A two-stage model for multiple target detection in rapid serial visual presentation. J. Exp. Psychol. Human Percept. Perform. 21, 109-127 (1995).

22. Broadbent, D. \& Broadbent, M. From detection to identification: response to multiple targets in rapid serial visual presentation. Percept. Psychophys. 42, 105-113 (1987).

23. Arnell, K. \& Jolicoeur, P. The attentional blink across stimulus modalities: evidence for centra processing limitation. J. Exp. Psychol. Human Percept. Perform. 25, 630-648 (1999).

24. Potter, M. C., Chun, M. M., Banks, B. S. \& Muckenhoupt, M. Two attentional deficits in serial target search: the visual attentional blink and an amodal task-switch deficit. J. Exp. Psychol. Human Percept. Perform. 24, 979-992 (1998).

25. Proksch, J. \& Bavelier, D. Changes in the spatial distribution of visual attention after early deafness. J. Cogn. Neurosci. 14, 1-5 (2002).

Supplementary Information accompanies the paper on www.nature.com/nature.

Acknowledgements We thank T. Monacelli, D. McColgin, J. Cohen and K. Schneider for help with subjects and software support; and R. Aslin, A. Pouget and D. Knill for feedback on the manuscript. This research was supported by the NIH and the James S. McDonnell-Pew Foundation.

Competing interests statement The authors declare that they have no competing financial interests.

Correspondence and requests for materials should be addressed to D.B.

(daphne@cvs.rochester.edu).

\section{Mitochondrial membrane remodelling regulated by a conserved rhomboid protease}

\section{G. Angus McQuibban, Saroj Saurya \& Matthew Freeman}

\section{MRC Laboratory of Molecular Biology, Hills Road, Cambridge CB2 2QH, UK}

Rhomboid proteins are intramembrane serine proteases that activate epidermal growth factor receptor (EGFR) signalling in Drosophila $^{1}$. Rhomboids are conserved throughout evolution ${ }^{2-5}$, and even in eukaryotes their existence in species with no EGFRs implies that they must have additional roles. Here we report that Saccharomyces cerevisiae has two rhomboids, which we have named $R b d 1 p$ and $R b d 2 p . R B D 1$ deletion results in a respiratory defect; consistent with this, Rbdlp is localized in the inner mitochondrial membrane and mutant cells have disrupted mitochondria. We have identified two substrates of Rbd1p: cytochrome $c$ peroxidase $(\mathrm{Ccp} 1 \mathrm{p})$; and a dynamin-like GTPase $(\mathrm{Mgm} 1 \mathrm{p})$, which is involved in mitochondrial membrane fusion $^{6-10}$. Rbd1p mutants are indistinguishable from Mgm1p mutants, indicating that Mgm1p is a key substrate of Rbdlp and explaining the $r b d 1 \Delta$ mitochondrial phenotype. Our data indicate that mitochondrial membrane remodelling is regulated by cleavage of Mgm1p and show that intramembrane proteolysis by rhomboids controls cellular processes other than signalling. In addition, mitochondrial rhomboids are conserved throughout eukaryotes and the mammalian homologue, PARL ${ }^{11}$, rescues the yeast mutant, suggesting that these proteins represent a functionally conserved subclass of rhomboid proteases.

Despite their widespread conservation, the only known function of eukaryotic rhomboid proteases is the activation of EGFR signalling in Drosophila ${ }^{12-15}$. We therefore examined their function in $S$. cerevisiae, which has no receptor tyrosine kinases but has two genes encoding rhomboids, which we have named RBD1 (YGR101w) and RBD2 (YPL246c). Deletion of RBD1 ( $\Delta r b d 1)$ caused cells to grow slowly (Fig. 1a), whereas deletion of $R B D 2$ had no obvious phenotype and did not enhance the phenotype of $r b d 1 \Delta$ cells (data not shown). The rbd1 $\Delta$ phenotype was rescued by plasmid-borne expression of RBD1 (Fig. 1a), confirming that the slow growth was indeed caused by loss of RBD1.

Although previously unnoticed, the Rbd1p sequence contains a signature motif for mitochondrial localization, as predicted by MitoProt ${ }^{16}$ and other algorithms. To test this prediction, we used homologous recombination to replace the wild-type gene with a fully functional gene fused at its carboxy terminus to green 